\title{
COMUNICACIÓN POLITICA, ELECCIONES Y DEMOCRACIA: LAS CAMPAÑAS DE DONALD TRUMP Y JAIR BOLSONARO
}

\author{
Dr. Arthur Ituassu \\ Pontificia Universidad Católica de Rio de Janeiro, Rio de Janeiro, Brasil \\ ituassu@puc-rio.br \\ ORCID iD: https://orcid.org/0000-0003-4781-1946 \\ Mg. Letícia Capone \\ Pontificia Universidad Católica de Rio de Janeiro, Rio de Janeiro, Brasil \\ leticiacapone@gmail.com \\ ORCID iD: https://orcid.org/0000-0003-3134-6701 \\ Mg. Leonardo Magalhães Firmino \\ Pontificia Universidad Católica de Rio de Janeiro, Rio de Janeiro, Brasil \\ leonardo_firmino@msn.com \\ ORCID iD: https://orcid.org/0000-0002-7177-6944 \\ Mg. Vivian Mannheimer \\ Pontificia Universidad Católica de Rio de Janeiro, Rio de Janeiro, Brasil \\ vmannheimer@gmail.com \\ ORCID iD: https://orcid.org/0000-0002-4072-8755 \\ Mg. Felipe Murta \\ Pontificia Universidad Católica de Rio de Janeiro, Rio de Janeiro, Brasil \\ femurpi@gmail.com \\ ORCID iD: https://orcid.org/0000-0001-9778-8444
}

Recibido el 14 de diciembre de 2018

Aceptado el 14 de octubre de 2019

\section{Resumen}

Este artículo tiene como objetivo reflexionar acerca de los posibles impactos de prácticas de comunicación política digital en el ambiente electoral sobre la democracia brasileña y las democracias en general, a partir de las elecciones presidenciales en Estados Unidos (2016) y Brasil (2018). Nuestro propósito es desarrollar una discusión en el marco de la "americanización", "modernización", "posmodernización" e "hipermediatización" de las campañas, así como a través de la idea de "eclipse del público" de John Dewey (1927). En perspectiva comparada, realizaremos una breve contextualización de las dos elecciones, con un enfoque especial en las plataformas de Donald Trump, en los Estados Unidos y de Jair Bolsonaro, en Brasil. Las mismas serán estudiadas con base en una metodología de estudio de caso en perspectiva histórica, mediante la cual tres 
investigadores analizaron más de 300 documentos, incluyendo artículos, reportajes, informes, entrevistas, etc. El análisis del contenido fue realizado a partir de la idea de "propaganda computacional", como una práctica comunicacional que utiliza datos, algoritmos y automatización para administrar y diseminar información (y desinformación) en los medios digitales. A partir de las contextualizaciones y discusiones propuestas, sugerimos: 1) una noción más amplia de "hipermediatización" como paradigma de entendimiento de los procesos de comunicación política en los contextos electorales, lo que llamamos de "hipermediatización extendida"; y 2) la idea de "eclipse del público", de John Dewey, para evaluar los problemas potenciales generados en la esfera pública por la fragmentación del público en varios minipúblicos. Con lo expuesto anteriormente, esperamos contribuir para los debates sobre cómo las tecnologías digitales de la comunicación pueden afectar, no solamente a las campañas, sino también a la política y a la democracia.

Palabras clave: internet y democracia, comunicación política comparada, campañas electorales online, Donald Trump, Jair Bolsonaro. 


\title{
POLITICAL COMMUNICATION, ELECTIONS, AND DEMOCRACY: THE CAMPAIGNS OF DONALD TRUMP AND JAIR BOLSONARO
}

\begin{abstract}
This article aims to discuss possible impacts of digital political communication on Brazilian democracy, and democracy in general, considering the 2016 presidential elections in the United States and 2018, in Brazil. We propose a discussion within the frameworks of "Americanization", "modernization", "post-modernization" and "hypermediatization" of the campaigns, and in relation to John Dewey's idea of "the eclipse of the public" (1927). In this sense, we make a brief contextualization of both elections focusing especially on the successful campaigns of both Donald Trump in the United States and Jair Bolsonaro in Brazil. Both campaigns were analyzed as historical case studies, and more than 300 documents, including articles, news stories, reports and interviews, among other sources were read and analyzed by three distinct researchers. A content analysis was developed by using the notion of "computational propaganda" as a reference, looking for practices that use data, algorithms, and automation to deliberately manage and disseminate information (and disinformation) in the digital media. Following both contexts and discussions, we suggest: 1) a larger notion of "hypermediatization" as a framework for understanding digital political communication practices in electoral contexts, what we have called "expanded hypermediatization"; and 2) John Dewey's idea of "the eclipse of the public" to evaluate potential problems to the public sphere raised by the fragmentation of the public into multiple minipublics. In the end, we hope to contribute to the debates about how digital media may affect not only campaigns and elections, but also politics and democracy.
\end{abstract}

Keywords: Internet and democracy, comparative political communication, online electoral campaigns, Donald Trump, Jair Bolsonaro. 
Introducción

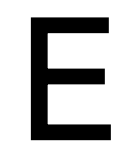

n una línea ya tradicional de estudios sobre campañas electorales online en Brasil y en el mundo (Bimber y Davis, 2003; Bimber, 2014; Braga, 2014; Braga, Carlomagno, 2018; Cervi, Massuchin, y Carvalho, 2016; Gomes y Aggio, 2009; Gomes et al, 2009; Graham et al., 2013; Ituassu et al., 2014; Ituassu et al, 2018; Marques et al., 2013; Muniz et al., 2016; Murta et al, 2017; Nielsen y Vaccari, 2013; Norris, 2000; 2002; Strommer-Galley, 2000, 2019; Vaccari, 2008), el objetivo de este artículo es producir una reflexión acerca de posibles impactos de la comunicación política digital sobre la democracia, tras las elecciones presidenciales de 2016 en los Estados Unidos, y de 2018 en Brasil. De manera comparada (Blumler y Gurevitch, 1995; Blumler y Kavanagh, 1999; Canel y Voltmer, 2014; Esser y Pfetsch, 2004; 2016; Hallin y Mancini, 2004a; 2011; Norris, 2009; Voltmer, 2006; Zielonka, 2015), se trata de un trabajo de contextualización de ambas contiendas respecto a la comunicación política digital de las campañas de Donald Trump y Jair Bolsonaro, discutiendo tales contextos a partir de los conceptos de "americanización", "modernización", "posmodernización" e "hipermediatización". Además, en el campo de la teoría de la democracia, sugerimos una posible relación entre la comunicación política de las campañas con la idea de "eclipse del público" de John Dewey (2012 [1927]).

En 2016, la elección presidencial en Estados Unidos fue marcada por la polémica plataforma de Donald Trump, centrada en Facebook y acusada de haber desarrollado prácticas de desinformación y estrategias ilegales de big data (Cadwalladr y Graham-Harrison, 2018). Además, fue una campaña notoria por la influencia extranjera (Persily, 2017; Intelligence Community Assessment, 2017). En 2018, en Brasil, la elección presidencial también fue caracterizada por controversias. Jair Bolsonaro centró su campaña en los medios sociales, especialmente en WhatsApp y en Facebook. Alrededor de la campaña, fueron detectadas prácticas de desinformación y automatización (Arnaudo, 2019; Ruediger, 2018c, 2018d).

En la primera sección de este artículo, discutiremos las nociones de "americanización" y "modernización" de las campañas y los avances históricos de la comunicación política online durante las elecciones. A seguir, en las secciones 2 y 3 analizaremos los contextos electorales estadounidense y brasileño. En relación a las campañas de Trump y Bolsonaro, optamos por una metodología de estudio histórico de caso (King, Keohane y Verba, 1994; Norris, 2010; Strommer-Galley, 2019). En ambos casos, utilizamos fuentes digitales como informes, artículos, noticias, entrevistas, etc., recogidas en la web antes, durante y después de las elecciones de 2016 y 2018. Más de 300 documentos sobre ambas campañas fueron seleccionados y analizados por tres investigadores distintos. En este material buscamos cualquier señal de propaganda computacional, definida por Woolley y Howard como un término que encuadra ese reciente fenómeno - y el 
campo de estudio emergente - de desinformación y maniobra digital. Como una práctica comunicativa, la propaganda computacional describe el uso de algoritmos, automatización y curaduría humana para gestionar y distribuir información y desinformación en los medios sociales digitales (Woolley, Howard, 2019).

En ambos procesos electorales, buscamos indicios de desinformación, noticias falsas, utilización de robots, big data, influencia externa y estrategia de medios sociales, en general. De esta forma, esperamos haber señalado evidencias suficientes para discutir, en la sección 4, al menos tres cuestiones sobre posibles impactos de las campañas digitales en la(s) esfera(s) pública(s), en el sistema de comunicación política y en el contexto electoral brasileño y en general. Por lo tanto, en las conclusiones sugerimos: 1) una noción más amplia de "hipermediatización" como paradigma de entendimiento de los procesos de comunicación política en los contextos electorales, lo que llamamos de "hipermediatización extendida"; y 2) la idea de "eclipse del público", de John Dewey, para evaluar algunos problemas potenciales generados en la esfera pública por la fragmentación del público en varios minipúblicos.

\section{Americanización, modernización, posmodernización...}

La "americanización" es una hipótesis tradicional de los estudios de comunicación política. Como afirman Swanson y Mancini (1996), dicha hipótesis sostiene que las campañas electorales en las democracias alrededor del mundo se vuelven cada más americanizadas, a medida que los candidatos, los partidos políticos y los medios de comunicación siguen las prácticas de sus pares en los Estados Unidos. Los autores sugieren que la hipótesis es un punto de partida útil para comparar las prácticas de campaña en diferentes países.

Puesto que la noción de americanización denota una excesiva influencia estadounidense en otros contextos, la idea de modernización se propuso como alternativa, en un esfuerzo por enfatizar que los cambios en la comunicación política no son generados solo por fuerzas exógenas, sino también arraigadas en procesos de cambios sociales (Hallin y Mancini, 2004b). Al mencionar a los principales actores políticos de un "sistema de comunicación política" (Blumler y Gurevitch, 1995; Gurevitch y Blumler, 2004; Howard, 2006), Pipa Norris (2002) sugiere que los cambios en la comunicación de campaña pueden ser mejor entendidos como un proceso evolutivo de modernización que simultáneamente transforma las prácticas de organización de campaña, de los medios de comunicación y del electorado. La autora clasifica tres tipos de campañas: premodernas, modernas y posmodernas. En lugar de afirmar que todas las campañas se desplazan hacia la categoría posmoderna, Norris enfatiza que los cambios deben entenderse como parte del proceso de modernización arraigado en desarrollos tecnológicos y políticos comunes a muchas sociedades postindustriales. 
En síntesis, la campaña premoderna se organiza sobre la base de formas directas de comunicación interpersonal entre candidatos y ciudadanos a nivel local. El electorado y la prensa están anclados por una fuerte lealtad partidista, donde esta actúa como mediadora principal entre los partidos y el público. Como Howard (2006) resumió, este es el tipo de campaña en la que voluntarios locales del partido toman conciencia de las posiciones de los miembros de la agremiación con encuentros partidarios y esfuerzos de prospección en el ámbito local. En esta tipología de campaña existe también un bajo nivel de control centralizado de la logística, es decir, las secciones locales de los partidos seleccionan a los candidatos, administran las campañas, publican los panfletos, gestionan los recursos y proveen al engranaje que conecta los electores a los candidatos (Norris, 2002).

Las campañas modernas están marcadas por la televisión y las encuestas de opinión y se definen por tener una organización más coordinada por los líderes políticos centrales, asesorados por consultores profesionales externos. Para este tipo de campaña, la televisión se convierte en el principal medio para alcanzar al electorado. Los políticos y los consultores profesionales conducen investigaciones, diseñan anuncios, programan el tema del día, los viajes de campaña, las ruedas de prensa y las oportunidades de fotos, al tiempo que luchan por dominar los noticieros y espacios de mayor audiencia en la televisión. En las campañas modernas, los noticieros del horario estelar (prime time) de televisión son el medio más importante para divulgar la campaña (Howard, 2006).

En las campañas posmodernas los consultores profesionales de publicidad, marketing y opinión pública asumen un papel más central no solo en los momentos electorales, sino también después de las elecciones, con el gobierno actuando en campaña permanente. Los medios periodísticos son más fragmentados en un entorno más complejo de múltiples canales y el electorado se vuelve más flexible en sus elecciones. Las campañas posmodernas intentan manipular a la opinión pública no solo con el propósito de ganar elecciones, sino también de legitimar la gobernanza (Norris, 2002).

Howard, siguiendo la clasificación de Norris, presenta un cuarto tipo de campaña, nombrada por él como campaña hipermedia, más fugaz que permanente. El atributo más importante de este tipo de campaña son los datos, especialmente, los que contribuyen a las estrategias de comunicación política. En el contexto de la campaña hipermedia, los perfiles de los votantes, de los donantes, de los voluntarios y de los candidatos, así como otras informaciones estratégicas, son utilizados por los consultores en la formulación y diseminación de la comunicación política (Howard, 2006).

Para el autor, las campañas hipermedias se desarrollan con los nuevos medios digitales, con anuncios de campaña dirigidos, creados y distribuidos a través de medios digitales. En este contexto, las personas que antes podían solamente 
consumir el contenido político, pasan a poder producirlo y distribuirlo por su cuenta. Si las campañas de medios masivos difunden contenido producido por las consultorías para un gran número de personas, las campañas hipermedias restringen el contenido a las personas deliberadamente seleccionadas y el contenido en sí se ajusta a los datos del público objetivo (Howard, 2006). Por lo tanto, en el contexto de las campañas hipermedias, la propaganda computacional es descrita por Woolley y Howard (2019) como una práctica comunicativa con algoritmos, automatización y curaduría humana para administrar y distribuir desinformación en las redes digitales. De esta forma, a partir de las nociones de modernización o americanización de las campañas, se puede pensar también en una "hipermediatización", ligada a los desarrollos tecnológicos digitales accesibles a una amplia gama de usuarios y sociedades en todo el mundo. Un proceso de hipermediatización que permite que las campañas descubran y dirijan a la opinión pública, a la vez que crean minorías (en lugar de seguir mayorías), administrando el desarrollo contemporáneo de la ciudadanía (Howard, 2006).

\section{Estados Unidos, 2016: datos, Facebook y fake news}

No hay duda de que, en relación con las campañas de Barack Obama de 2008 y 2012, la elección presidencial de 2016 en los Estados Unidos ha traído un cambio claro de expectativas en lo que se refiere a los impactos en la democracia de las tecnologías digitales de comunicación, al punto de que un comentador preguntó en un importante periódico: "¿Puede la democracia sobrevivir a Internet?" (Persily, 2017). Es importante recordar que las campañas demócratas de 2008 y 2012 establecieron un nuevo nivel en las relaciones entre Internet y las elecciones, anunciando la llamada tercera fase del campo, marcada por los anuncios en línea y el uso ampliado de los medios sociales (Davis et al., 2009).

La campaña de Obama en 2008 estuvo presente en más de diez medios sociales, incluyendo Facebook, MySpace, Twitter y Black Planet y tuvo al menos tres canales en YouTube (Fox, 2012). Además, la plataforma demócrata creó sus propios medios sociales, MyBO, donde se resaltaban, en los perfiles de los usuarios, las características relacionadas a la movilización política en la campaña (Gomes et al., 2009, Fox, 2012). En términos de recaudación, la campaña de Obama en 2008 llegó a unos US\$ 750 millones, frente a los US\$239 millones de su rival republicano, John McCain. Un comentarista sugiere que más de US\$ 400 millones del total recaudado por Obama se debió al esfuerzo en la esfera digital. Casi 4 millones de estadounidenses hicieron alguna donación a la campaña demócrata en 2008 (Scherer, 2012). Cuatro años después la misma estrategia fue desarrollada, pero en un contexto mucho más favorable. En el año 2012, cerca del $70 \%$ de la población en los Estados Unidos utilizaba algún tipo de medio social. En 2008, eran solamente 37\% (Rutledge, 2013).

Por otro lado, en 2016, la campaña victoriosa de Donald Trump incluyó una serie de prácticas y cuestiones que propiciaron una cierta inversión de expectativas. De hecho, uno de los aspectos más importantes de este proceso fue la dirección 
estratégica de los medios sociales en la campaña, marcada por la elección polémica de Brad Parscale, experto en marketing digital y un outsider en el mundo de la consultoría política estadounidense, considerado el primer consultor "puramente digital", a dirigir una importante campaña presidencial en los Estados Unidos (Persily, 2017).

La estrategia se centró en la importancia de Facebook, incluso sobre los anuncios televisivos. Mientras la campaña de Hillary Clinton gastó US\$258 millones en anuncios en la televisión, la plataforma Trump invirtió US\$ 100 millones, menos de la mitad respecto a la primera. En Facebook, se estima que el equipo de Parscale fue capaz de difundir hasta 100 tipos diferentes de anuncios dirigidos en tan solo 24 horas. Un dato polémico del sistema desarrollado, sin embargo, se refiere al uso de los llamados dark posts, que son publicaciones que no aparecen en la página oficial de la campaña, pero sí para quienes fueron enviados. En una entrevista, Parscale afirma, como ejemplo, que podría enviar en forma de dark posts una serie de informaciones negativas sobre la infraestructura en el gobierno democrático a electores de un pequeño condado, preocupados por la infraestructura y de voto tradicionalmente liberal (CBS, 2017).

A partir del momento en que un dark post pasa a ser compartido en los medios sociales, resulta muy difícil identificar su origen. Con los dark posts, la campaña adquiere más libertad para trabajar con contenidos negativos y discursos para públicos específicos, sin que otros grupos se enteren de la publicación o puedan asociarla a la campaña. Esto puede haber sido particularmente importante en las elecciones de 2016 con los mensajes enviados a grupos de votantes más propensos a apoyar a los demócratas, como mujeres, negros y trabajadores de la industria, con el propósito de desmovilizarlos a votar en Hillary Clinton (Delany, 2017).

Una estimación apunta a que los mensajes de la campaña de Trump fueron tres veces más retuiteados y cinco veces más compartidos en Facebook que aquellos vinculados a la plataforma adversaria (Persily, 2017). De hecho, investigaciones apuntan a que la plataforma de Trump ha hecho un uso intensivo de automatización, en busca de los llamados "consensos artificiales", generados por la amplificación automatizada del tráfico en la red alrededor de un candidato o cuestión. Según el informe del Oxford Internet Institute (OII), la proporción de los mensajes automáticos de Trump con relación a las disparadas por la campaña de Hillary Clinton fue de 5 a 1 (Woolley y Guilbeault, 2017).

Como afirman Woolley y Guilbeault, las campañas, los candidatos y los usuarios utilizan robots en las elecciones hace casi una década, pero la campaña estadounidense del 2016 fue un hito en el uso de automatización política y propaganda computacional en el ambiente electoral del país. En base a una muestra de 17 millones de tweets, los autores demuestran cómo los bots fueron 
capaces de ocupar posiciones centrales en la mediación de la información en Twitter durante las elecciones (Wooley, Guilbeault, 2019).

En ese contexto, además de Parscale, otro personaje central de la campaña de Donald Trump fue Steve Bannon, que trabajó con la empresa Cambridge Analytica, responsable de la estrategia de datos de la campaña de Donald Trump. Bannon es un ejecutivo de medios en Estados Unidos y consultor político que dirigió el sitio de noticias de extrema derecha Breitbart News, fundada en 2007 por el comentarista conservador Andrew Breitbart. Como es sabido, Cambridge Analytica fue cerrada tras el escándalo de filtración de información personal de 50 millones de ciudadanos estadounidenses en Facebook (Cadwalladr y GrahamHarrison, 2018). Nacida de un modelo generado en la Universidad de Cambridge, la empresa almacenaba los perfiles en grandes bancos de datos $y$, por medio de ellos, ofrecía el psychological targeting de cada usuario (Grassegger y Krogerus, 2017). Creada en 2013, Cambridge Analytica ya había participado en 44 campañas en los Estados Unidos en 2014 y en junio de 2016 fue contratada por la campaña de Trump.

La polémica agencia de datos llegó a confirmar, en 2017, un acuerdo de sociedad con una empresa brasileña para actuar en Brasil. El acuerdo dio origen a la firma CA-Ponte y tenía como foco "la transferencia y la 'tropicalización' de la metodología de segmentación psicográfica" (Mota, 2017). Después del escándalo de la fuga de datos y del posterior cierre de Cambridge Analytica, el negocio no se desarrolló, por lo menos no oficialmente. En agosto de 2018, Eduardo Bolsonaro, hijo de Jair Bolsonaro, publicó una foto en la red Instagram sobre un encuentro con Steve Bannon, en Nueva York (Brito, 2018). Sobre el encuentro, Eduardo Bolsonaro afirmó que Bannon se mostró un "entusiasta de la campaña de Bolsonaro", añadiendo: "Ciertamente estamos en contacto para sumar fuerzas" (De Sá, 2018).

Además de la estrategia de datos, otro elemento importante en las elecciones de 2016 en Estados Unidos fueron las noticias falsas (fake news). Entre los casos más notorios están el del "pizzagate", que se refiere al dueño de una pizzería y recaudador de fondos del partido Demócrata, James Alefantis, que fue falsamente acusado de pedofilia (Ruediger, 2018c, Delmazo y Valent, 2018). Otro caso emblemático fue el del supuesto apoyo del Papa Francisco a Donald Trump, que fue divulgado por la página web WTOE 5. Dicha publicación, quizás la fake news más representativa de ese momento, fue compartida más de un millón de veces (Allcott y Gentzkow, 2017). En las elecciones de 2016, en Estados Unidos, las noticias falsas aparecieron ligadas preponderantemente a los medios sociales. Allcott y Gentzkow, por ejemplo, calculan que estos medios fueron el origen de más del $40 \%$ de los accesos a los sitios de fake news durante la contienda. Además, se estima que hubo por lo menos 40 millones de noticias falsas compartidas en Facebook durante esas elecciones, con tres veces más noticias falsas pro-Trump que pro-Clinton. De la misma forma, las noticias falsas pro- 
Trump fueron cuatro veces más compartidas que las pro-Clinton (Allcott y Gentzkow, 2017).

Por último, sobre la influencia externa vía internet, hubo varios ejemplos de ese fenómeno en las elecciones de 2016 en Estados Unidos. Dos casos notorios son el de los jóvenes de la ciudad de Veles, en Macedonia, y el de la interferencia rusa. En el primer ejemplo, un grupo de adolescentes descubrió que podría ganar dinero publicando historias pro-Trump y anti-Clinton en los casi 140 sitios de noticias falsas que manejaban. A medida que las noticias falsas se volvían más ultrajantes, las páginas atraían a más usuarios, que podrían hacer clic en los anuncios de publicidad (Google Ads) instalados en los sitios web. Cuantos más clics estos anuncios recibían, más ganancias generaba el sistema (Allcott, Gentzkow, 2017; Persily, 2107). En el segundo caso, un enorme debate se estableció en Estados Unidos sobre la actuación de Moscú en las elecciones de 2016. En ese contexto, hay indicios de propaganda producida y difundida vía internet, especialmente en los medios sociales, con la actuación de agencias de medios de comunicación del gobierno ruso o subsidiadas por el Estado (Persily, 2017, Intelligence Community Assessment, 2017).

\section{Brasil, 2018: WhatsApp y automatización}

En Brasil, el uso de los medios digitales en campañas electorales no es reciente (Braga y Carlomagno, 2018), así como no lo es el uso de robots o noticias falsas, que no representaron novedades en las elecciones de 2018 y ya aparecieron en contiendas o eventos políticos anteriores, como las manifestaciones de 2013 o en el caso del asesinato de la concejal de la ciudad de Rio de Janeiro, Marielle Franco (Arnaudo, 2017; Ruediger, 2017, 2018a, 2018b). Como afirman Braga y Carlomagno, a partir de 2010 empieza una segunda fase de las campañas online en Brasil, caracterizada por la " disminución de las restricciones legales" y por el "uso creciente de diferentes recursos digitales" (Braga y Carlomagno, 2018, p.15).

En este contexto, según un estudio del Directorio de Análisis de Políticas Públicas (DAPP), de la Fundación Getúlio Vargas, los robots fueron responsables de cerca del $10 \%$ de las interacciones en Twitter durante la campaña para las elecciones de 2014 (Ruediger, 2018b). Específicamente, en el momento del debate en la Red Globo de Televisión, entre los entonces candidatos Aécio Neves (PSDB) y Dilma Rousseff (PT), en la segunda vuelta, el trabajo sugiere que casi el $20 \%$ de las interacciones favorables a Aécio Neves en Twitter fueron causadas por la acción de robots. Otro informe del Oxford Internet Institute (OII), identificó que los hashtags relacionados con el candidato del PSDB triplicaron después de 15 minutos de debate transmitido por televisión. Según el texto, las campañas de Dilma y Aécio usaron robots, pero la plataforma del PSDB lo hizo a una escala mucho mayor, llegando a gastar hasta R\$10 millones en el desarrollo de cuentas automatizadas en Twitter, Facebook y también en WhatsApp (Arnaudo, 2017). Además, un punto resaltado en ambos informes es la continuidad de uso de cuentas automatizadas por parte del PSDB, contra el gobierno de Rousseff $y$, 
poco a poco, a favor del impeachment de la presidenta, llevado a cabo por el Congreso Nacional brasileño entre 2015 y 2016. Otro informe del DAPP, de marzo de 2018, indicó también la presencia de robots de origen ruso en la diseminación de material de campaña de Aécio Neves en 2014 (Ruediger, 2018b). Dos años después, en 2016, año de elecciones locales en el país, un estudio realizado por la empresa multinacional de ciberseguridad Symantec colocó a Brasil en octavo lugar en el ranking de los países con mayor presencia de robots (Symantec, 2016).

En 2018, Brasil llegó al contexto electoral con más de 110 millones de usuarios de internet, ocupando el tercer lugar entre los países con mayor número de usuarios de Facebook y el sexto, entre los usuarios de Twitter (Ruediger, 2018c). Además, para el año 2018, las restricciones legales a las campañas online se redujeron aún más, en función de las nuevas reglas editadas por el Congreso Nacional en 2017 (Ley 9.504/97), que pasaron a permitir el llamado "impulso de contenido", como forma de propaganda pagada en internet (Brito y Massaro, 2018a; 2018b). En ese contexto, según Arnaudo (2019), la propaganda computacional en forma de robots actuando en red, fake news y manipulación por medio de algoritmos pasaría a desempeñar un papel central en el sistema político brasileño y, de hecho, la comunicación política digital de la campaña victoriosa de Jair Bolsonaro presenta elementos claros de propaganda computacional.

En un informe publicado durante las elecciones, entre la primera y la segunda vuelta de las presidenciales, las interacciones provocadas por robots en Twitter llegaban al 10,4\%, con un 13,8\% entre los partidarios de Bolsonaro. Los robots en favor del candidato movieron el 70,7\% de las interacciones automatizadas en Twitter en aquel periodo. En ese punto de la elección, había un promedio de 1,5 millones de tweets por día sobre los candidatos. Entre el 10 y el 16 de octubre, hubo 852,3 mil publicaciones de robots, de las cuales 602,5 mil desde la base de apoyo de Bolsonaro (Ruediger, 2018d).

Respecto a las noticias falsas en el contexto brasileño, las mismas aparecen más ligadas a WhatsApp que a Facebook, como fue el caso de las elecciones de 2016, en Estados Unidos. En este contexto, un monitoreo hecho por el diario El País, en septiembre de 2018, acerca de las posturas en tres grupos públicos de WhatsApp favorables a Jair Bolsonaro, muestra que dos de ellos distribuían desinformación de forma evidente. En esa ocasión había al menos 100 grupos públicos de WhatsApp a favor de dicho candidato (Benites, 2018).

Entre los casos que se hicieron públicos aún en el período electoral, están el supuesto fraude en las urnas electrónicas (Mota, 2017); las llamadas de Manuela D'Ávila (PCdoB), candidata a vicepresidenta en la lista de Fernando Haddad (PT), a Adélio Bispo de Oliveira, el responsable por el haber apuñalado a Bolsonaro en plena campaña (Beraldo, 2018); la denuncia de agresión a una señora por ser electora de Bolsonaro (Barragán, 2018); la acusación de que el candidato 
Fernando Haddad habría hecho apología al incesto en uno de sus libros (Barragán, 2018); la acusación de Joice Hasselmann, en ese entonces candidata a diputada federal, de que un importante órgano de prensa habría recibido $\mathrm{R} \$ 600$ millones para atacar la candidatura de Jair Bolsonaro (Benites, 2018); el texto divulgado por Eduardo Bolsonaro, hijo de Jair Bolsonaro, donde indica que el grupo "Mujeres Unidas Contra Bolsonaro" en Facebook habría comprado su base de más de un millón de usuarios de una página cualquiera ya existente (Becker, 2018); y, finalmente, el polémico caso sobre el "kit gay" (Barragán, 2018). Sobre esta última noticia, en particular, el propio Jair Bolsonaro presentó en una entrevista televisiva, el libro que en Brasil se intitula "Aparelho Sexual e Cia - Um guia inusitado para crianças descoladas" (título original en francés: "Le Guide du zizi sexuel"), escrito por Hélène Bruller e ilustrado por ZEP (Philippe Chappuis), afirmando falsamente que el mismo había sido distribuido en las escuelas públicas brasileñas (Salgado, 2018).

En Facebook, el monitoreo sugiere que, en Brasil, no se utilizó una táctica de propaganda como la de la campaña de Donald Trump, en 2016, con el envío de publicidad micro direccional. No hay indicios de una gran campaña de anuncios pagados por la candidatura Bolsonaro, ni siquiera acciones relevantes de micro dirección (Brito Cruz, Massaro, 2018b). En realidad, el político llegó a las elecciones de 2018 con una posición consolidada en Facebook, obtenida a lo largo de años, y muy superior a la de sus adversarios.

Así, por ejemplo, en base a la compilación de datos realizada en el 2014, sobre el número de publicaciones y la media de interacciones en Facebook de ocho diputados federales del estado de Rio de Janeiro durante sus campañas, Jair Bolsonaro presentó, en ese entonces el promedio de interacciones (likes, comentarios y número de comparticiones) 29 veces mayor respecto al segundo colocado en la lista, con casi 30 mil interacciones por publicación (Murta et al, 2017). Durante las elecciones de 2018, la campaña de Jair Bolsonaro logró alcanzar en una única transmisión un pico de 1,3 millón de interacciones y 6,6 millones de visualizaciones (Ruediger, 2018c).

Sin embargo, a diferencia del contexto estadounidense en 2016, cuando Facebook fue el centro de la campaña de Donald Trump, el WhatsApp parece haber alcanzado una relevancia mayor en Brasil en 2018. Como explica Brito y Valente (2018), ya que se trata de una aplicación de los mensajes cifrados, el WhatsApp no ofrece ni publicidad, ni la posibilidad de dirección - el mensaje solo se disemina si alguien lo retransmite a otros (o si alguien lo envía masivamente, lo que choca con la legislación electoral, que prohíbe el envío de mensajes a números de teléfono que no fueron voluntariamente informados por sus propietarios durante las campañas). En red, esta infraestructura de propaganda se vuelve eficiente "cuando los administradores de los grupos se coordinan, actuando como 'nudos' importantes de la red, por concentrar muchas conexiones" y "cuando militantes conectan a ella no solo su tiempo y redes 
personales, sino grandes recursos financieros y herramientas más sofisticadas". Con eso, en el WhatsApp la gestión de tal infraestructura parece contar con una mezcla de trabajo pagado y voluntario, con estructura descentralizada (Brito y Valente 2018). En ese contexto, el diario Folha de S. Paulo publicó el 18 de octubre de 2018, entre la primera y la segunda vuelta de la contienda electoral, que empresarios brasileños estaban financiando ilegalmente una campaña por WhatsApp, a favor de Bolsonaro, con contratos de hasta $\mathrm{R} \$ 12$ millones (Campos, 2018).

\section{Discusión: comunicación política digital y democracia}

Como ya fue mencionado, en este artículo tenemos la intención de sugerir: 1) una noción más amplia de "hipermediatización" como paradigma de entendimiento de los procesos de comunicación política en los contextos electorales; 2) la idea de "eclipse del público", de John Dewey, para evaluar algunos de los problemas potenciales generados por la fragmentación del público en varios minipúblicos. En este sentido, esta sección tiene el propósito de discutir estas dos sugerencias, relacionadas a los dos contextos electorales previamente presentados.

\subsection{Posmodernización, hipermediatización}

La propuesta relativa a la idea de hipermediatización como paradigma adecuado para el entendimiento de los desarrollos contemporáneos de la comunicación política en contextos electorales alrededor del mundo proviene de una acción de rescate de discusiones tradicionales del campo para el análisis de lo que viene ocurriendo más recientemente en los medios digitales. Este rescate se materializa a partir de una sensación de que una buena parte de los estudios enfocados en los medios digitales parece hoy más orientada hacia los aspectos estratégicos de la comunicación política electoral (Murta et al, 2017, Miola y Marques, 2018), sus énfasis (Sousa y Marques, 2017) o formas de uso de las herramientas y aplicaciones (Enli y Skogerbo, 2013, Muñiz et al, 2016), dejando de lado una tradición anterior más preocupada por los impactos de las relaciones entre los medios y las elecciones sobre el ambiente democrático (Blumler y Gurevitch, 1995; Swanson y Mancini, 1996; Le Duc, Niemi y Norris, 2002; Anstead y Chadwick, 2008; Gomes et al., 2009).

Como se mencionó anteriormente, Swanson y Mancini (1996) sugieren que la hipótesis de la "americanización" -es decir, que las campañas en las democracias alrededor del planeta estarían más y más "americanizadas", en la medida en que partidos políticos, candidatos y la cobertura periodística seguirían los desarrollos ocurridos en los Estados Unidos - es un provechoso punto de partida para el análisis comparado de las prácticas de campaña en países distintos (Swanson y Mancini, 1996). En este caso, la hipótesis sirvió también como punto de partida para las discusiones presentadas.

Aunque teniendo en cuenta el término "modernización", que vacía la noción incorporada en la idea anterior de que las transformaciones en las prácticas de 
campaña en las varias democracias del planeta tendrían una faceta preponderantemente exógena, con su centro original en Estados Unidos, y considerando, además, la sugerencia de Pipa Norris (2002) de que los cambios en las prácticas de campaña deben ser entendidos como parte del proceso de modernización de las sociedades, basado en los desarrollos tecnológicos y políticos, similares a los ocurridos en las sociedades postindustriales, la cuestión de la "americanización", a nuestro juicio, no pierde su importancia dado que muchos de estos desarrollos tecnológicos y políticos, en particular en lo que se refiere a las tecnologías digitales de comunicación, surgen primero en los Estados Unidos, como es el caso de la mayoría de las aplicaciones utilizadas hoy en campañas alrededor del mundo. Esto justifica la importancia que le damos en este artículo a las elecciones de 2016 vencidas por Donald Trump.

Sin embargo, los términos "americanización" y "modernización" están muy orientados a la diseminación de las llamadas prácticas modernas de campaña, relacionadas con las encuestas electorales, centralización estratégica y, principalmente, a buscar la atención del elector a través de la televisión. En ese sentido, una actualización del paradigma se hace necesaria, sobre todo para contemplar las transformaciones más recientes oriundas del uso de los medios digitales. Así, las propuestas de clasificación de las campañas por los términos "posmodernas" e "hipermedias" surgen como opciones. Como hemos visto, en las llamadas campañas "posmodernas", los consultores políticos de marketing y opinión pública asumen papeles centrales no solo durante el período electoral, sino también durante el gobierno, en lo que se ha convenido llamar "campaña permanente" (Norris, 2002). Las prácticas se desarrollan en un sistema de medios más complejo y fragmentado y el electorado es percibido como más flexible en su comportamiento. Según Norris (2002), las campañas permanentes intentan controlar a la opinión pública no solo para vencer las elecciones, sino también para legitimar la gobernanza.

Dado que el análisis aquí propuesto pone particular atención en las campañas electorales, a nosotros nos interesa el énfasis dado por Norris a un sistema mediático más complejo y fragmentado y un electorado más volátil. Ambos elementos pueden ser complementados con la noción de "hipermediatización" de las campañas, que, a diferencia de la idea de "posmodernización", se centra más en el período electoral y tiene como principal atributo el uso político y estratégico de datos. Como sugiere Howard (2006), las campañas hipermedia se desarrollan con los medios digitales, con anuncios dirigidos y distribuidos vía internet. En el contexto de esas campañas, el ciudadano que tradicionalmente consume contenido político, se vuelve también un potencial productor y diseminador. Una campaña hipermedia produce contenido seleccionado para audiencias seleccionadas y la propaganda computacional es uno de sus elementos principales. 
Con ello, este artículo sugiere, a partir de la noción tradicional de "americanización", una noción más amplia de "hipermediatización" de las campañas, la "hipermediatización extendida". Esta definición más amplia incluye aspectos de la idea de "posmodernidad" para llamar la atención sobre prácticas de comunicación política electoral y no-electoral dirigidas, con uso de datos del elector y automatizadas, propagando información y también desinformación en los medios digitales.

\subsection{El eclipse del público}

La importancia y la actualidad de la obra The Public and Its Problems: An Essay in Political Inquiry, de John Dewey, publicado por primera vez en 1927, para el contexto social y político contemporáneo tiene por base, como escribió Rogers, el hecho de que ese texto "es una de las más ricas reflexiones sobre el futuro de la democracia en la era de la comunicación de masa" (Rogers, 2012: 1). Para el comentarista, el libro, en diálogo con Opinión Pública, de Walter Lippmann (2010 [1921]), tiene como objetivo abordar dos cuestiones distintas pero relacionadas: 1) ¿qué roles los ciudadanos y los especialistas deben ejercer en el contexto de la modernidad compleja, para que se mantenga la dimensión de autogobierno asociada con la democracia?; y 2) ¿cuál debe ser el método más adecuado para que el público emerja de su eclipse en el contexto de la modernidad compleja y de modo que pueda cumplir su papel de autogobernanza?

Como sugieren las preguntas, la idea de "eclipse del público" es uno de los puntos principales de la obra aquí referenciada, que tiene como base la noción de que consecuencias indirectas, extensivas, prolongadas y serias, como resultado de las acciones conjuntas o del comportamiento interactivo, claman la formación de un "público", con el interés común de regular esas consecuencias. Sin embargo, la modernización de las sociedades, para Dewey, multiplicó, intensificó y complicó enormemente el alcance de las consecuencias indirectas, formando un inmenso conjunto consolidado de combinaciones, de base impersonal y no comunitaria, de modo que el público resultante no consigue autoidentificarse o autodistinguirse.

Por supuesto, Dewey, como un pragmático, parte de la experiencia histórica estadounidense, es decir, de una democracia que se formó a partir de bases comunitarias, algo muy diferente del contexto brasileño (y también latinoamericano) en el que el Estado y las democracias fueron un proyecto histórico por parte de la autoridad constituida (Pamplona y Stuven, 2010). Sin embargo, a partir de la teoría del Estado y la democracia de John Dewey (y también de otros autores como Rousseau), puede que este hecho sea visto como una dificultad más que la sociedad brasileña (y latinoamericana) enfrenta en su desarrollo democrático.

Para la discusión aquí propuesta, es fundamental entender la relación que Dewey establece entre el desarrollo tecnológico y democrático de las sociedades. "Invente el ferrocarril, el telégrafo, la manufactura y la concentración de la 
población en centros urbanos y alguna forma de gobierno democrático es humanamente inevitable", escribió John Dewey (2012 [1927]: 101), a partir de su noción de que consecuencias indirectas llevarían a la formación de un "público", entidad necesaria para la construcción de la institucionalidad (el Estado) a regular esas mismas consecuencias. De esta forma, ferrocarriles, viajes y transportes, el comercio, los correos, el telégrafo y el teléfono, así como los periódicos, generan "interacción e interdependencia". En este sentido, para el autor, la unidad del Estado moderno es posible gracias a las consecuencias de la tecnología empleada para facilitar la circulación de opiniones e información.

A partir de ese punto, sin embargo, John Dewey, con su noción de "eclipse del público", concordaría con el diagnóstico de Walter Lippmann sobre la complejidad excesiva de las sociedades modernas, discrepando solamente de este último con relación a los caminos para el fortalecimiento democrático en este escenario. Mientras que Dewey apostaba por la educación para la ciudadanía, Lippmann miraba a la importancia de la especialización y los límites a la participación, con un paradigma de cuño más elitista, casi como una reproducción moderna de la República (de reyes filósofos) de Platón.

Lo que se propone aquí es que las campañas hipermedia, lejos de ser agentes potenciadores, presentan un obstáculo más para la constitución del "público", en el sentido que Dewey le otorga al término. El problema se daría por la capacidad que tienen esas campañas de, como resalta Howard (2006), crear minorías, minipúblicos, a partir de discursos dirigidos a audiencias específicas, generando un tipo de atomización política adicional de la sociedad - la atomización del público. Como afirma el autor, mientras las campañas modernas utilizaban encuestas de opinión para sondear al público sobre lo que estaría pensando sobre un determinado tema o asunto, para a partir de esas informaciones moldear su comunicación o acción política (cuyo problema mayor sería, desde el punto de vista de la filosofía política, la idea de la "tiranía de la mayoría"), las campañas hipermedias están más preocupadas "en crear minorías, en vez de seguir mayorías, dirigir la opinión pública, en lugar de obedecerla, y gestionar el desempeño contemporáneo de la ciudadanía" (Howard, 2006: 204).

En los Estados Unidos, en 2016, la estrategia de envío de mensajes específicos a grupos seleccionados a través de Facebook, incluso con el uso de los llamados dark posts, fue notoria, como ya expuesto en este artículo. Según Delany (2017), la campaña de Donald Trump utilizó la tecnología para alcanzar fragmentos específicos del electorado con mensajes moldeados en función del perfil de los votantes, pudiendo alcanzar hasta 15 personas de un condado, cifra para quienes una campaña tradicional nunca invertiría en un anuncio de televisión. Sobre este punto, la estrategia de datos dirigida por la empresa Cambridge Analytica cumplió un papel fundamental, dado que era exactamente esa la especialidad anunciada por la firma: "individual psychological targeting". 
En Brasil, al parecer, no hubo en las elecciones de 2018, acciones relevantes de micro dirección que involucren a Facebook, pero la campaña digital por WhatsApp es, por la propia naturaleza de la aplicación, dirigida a públicos específicos, que se constituyen de forma cerrada y dialogan entre ellos, muchas veces, incluso expurgando los elementos vistos como extraños en aquel ambiente, como mostró el seguimiento hecho por el diario El País (Benites, 2018). La combinación de públicos cerrados de posicionamiento político semejante hablando entre ellos y compartiendo noticias o informaciones de veracidad dudosa, potencia los problemas ya discutidos hace algunos años por la literatura sobre echo chambers (Garrett, 2009; Quattrocococi, Scala y Sustein, 2016; Vaccari et al., 2016).

Con ello, argumentamos que la noción de "eclipse del público", de John Dewey (2012 [1927], presenta un marco bastante adecuado para reflexionar sobre posibles problemas generados a la democracia por la atomización política del público en minipúblicos independientes y cerrados en sí mismos. En nuestra opinión, pese a constituir un electorado, la unión de varios pequeños públicos independientes sirve como obstáculo para la constitución de un público con potencial capacidad de deliberar o utilizar públicamente la razón (como deliberación) en beneficio público, así como en torno a causas comunes, dificultando el desarrollo democrático de la comunidad.

No se trata de sugerir que la variedad de públicos podría ser negativa para la democracia. La cuestión, en realidad, no es tanto de "pluralidad" sino de "aislamiento". Los potenciales problemas generados en el proceso de constitución de minipúblicos por campañas hipermedias provienen del hecho de que el "uso público de la Razón" exige la publicidad de esta. Cuando los minipúblicos hablan entre sí y están cerrados en sí mismos la idea de "público" o de "uso público de la Razón" no sólo se vacía, sino que se acaba de constituir una imposibilidad.

\section{Conclusión}

Este artículo tuvo como objetivo producir una reflexión sobre posibles impactos de la comunicación política digital para la democracia brasileña (y para las democracias en general), a partir de las elecciones presidenciales de 2016, en Estados Unidos, y de 2018, en Brasil. Nuestra intención fue la de desarrollar esta discusión a partir de las nociones de "americanización", "modernización", "posmodernización" y "hipermediatización" de las campañas, así como a través de la idea de "eclipse del público" de John Dewey (2012 [1927]). En ese sentido, construimos una breve contextualización de las dos elecciones, con especial énfasis en las plataformas victoriosas de Donald Trump, en Estados Unidos, y de Jair Bolsonaro, en Brasil. Esto fue realizado sobre la base de una metodología de estudio de caso, en la que más de 300 documentos, incluyendo artículos, reportajes, informes, entrevistas, etc. fueron analizados a partir del concepto de "propaganda computacional", sugerido por Woolley y Howard (2019), como una 
práctica comunicacional que utiliza algoritmos, automación y curaduría humana para deliberadamente administrar y diseminar la desinformación en los medios digitales.

A partir de las dos contextualizaciones y de las discusiones propuestas, sugerimos: 1) una noción más amplia de "hipermediatización" como paradigma de entendimiento de los procesos de comunicación política en los contextos electorales; y 2) la idea de "eclipse del público", de John Dewey, para evaluar algunos de los problemas potenciales generados por la fragmentación del público en varios minipúblicos por parte de las campañas hipermedias.

Creemos, sin embargo, que algunos puntos necesitan todavía ser mencionados. En primer lugar, es enorme el volumen de informaciones que aún aparecen, en el momento de la producción de este artículo, sobre lo que ocurrió en las elecciones de 2016, en Estados Unidos, y de 2018, en Brasil. El panorama histórico y reciente producido aquí es un conjunto de datos e informaciones reunidos con el propósito de resaltar algún grado de desarrollo de la relación entre Internet y elecciones, que nos permita reflexionar sobre posibles cuestiones que esa misma relación suscitaría en los ambientes electorales y en las democracias contemporáneas en general, en el intento de pensar especialmente en las consecuencias para el contexto brasileño. En este sentido, vale comentar que este artículo es solo un paso más de un proyecto mayor que tiene por fin analizar las prácticas comunicativas de las campañas digitales en contextos latinoamericanos, a partir de las elecciones de 2016 en los Estados Unidos, con vistas a centrarse en otras contiendas políticas, además de la brasileña de 2018, como en los casos de México y Colombia.

Además, el hecho de analizar prácticas de comunicación digital en contextos electorales no significa afirmar que los medios digitales tienen el poder de definir los resultados de las elecciones. En los casos aquí analizados, se puede percibir una notoria influencia del periodismo profesional, más tradicional en la exposición pública de los candidatos, sea en la cobertura sobre las declaraciones de Donald Trump en Twitter, sea en la enorme exposición mediática que Jair Bolsonaro recibió tras ser acuchillado, mientras hacía campaña en la ciudad de Juiz de Fora en septiembre de 2018, lo que ciertamente tuvo gran impacto en el desarrollo posterior de la elección.

Por último, a nuestro juicio, este trabajo no debe interpretarse como una vuelta al momento "apocalíptico" de la teorización sobre Internet y política y/o Internet y democracia, fase superada en la literatura, que hoy se encuentra más madura, en un momento más descriptivo y calcado en estudios empíricos (Gomes, 2018). Para nosotros, los nuevos elementos aportados por la campaña de 2016 en los Estados Unidos, que también se hicieron visibles en las elecciones de 2018 en Brasil, se configuran como una preocupación para la democracia, pero no se superponen de forma sustitutiva a otras experimentaciones positivas de democracia digital con 
miras al perfeccionamiento de la transparencia pública, la participación ciudadana o la deliberación. En vez de reanudar perspectivas superadas o sacar conclusiones generalistas, preferimos reforzar la importancia de analizar caso por caso.

\section{Financiamiento}

Este trabajo ha sido realizado gracias al apoyo de la Coordenação de Aperfeiçoamento de Pessoal de Nível Superior - Brasil (CAPES) - Código de Financiación 001. 


\section{Referencias bibliográficas}

ALCOTT, H. y GENTZKOW, M. (2017): Social Media and Fake News in the 2016 Election. Journal of Economic Perspectives, V. 31 (2) pp. 211-236.

ANSTEAD, N. y CHADWICK, A. (2008): Parties, Election Campaigning and the Internet: Toward a Comparative Institutional Approach. In CHADWICK, A. y HOWARD, P. N. The Handbook of Internet Politics. New York, Routledge.

ARNAUDO, D (2019): Brazil: Political Bot Intervention During Pivotal Events. In WOOLLEY, S. y HOWARD, P. N. Computational Propaganda: Political Parties, Politicians, and Political Manipulation on Social Media. Nova York, Oxford University Press.

(2017): Computational Propaganda in Brazil: Social Bots during Elections. Oxford Internet Institute, Working Paper No. 2017.8, 2017. Disponible en: http://comprop.oii.ox.ac.uk/wp-content/uploads/sites/89/2017/06/CompropBrazil-1.pdf.

BARRAGÁN, A. (2018): Cinco 'fake news' que beneficiaram a candidatura de Bolsonaro. El País Brasil, 19/10. Disponible en: https://brasil.elpais.com/brasil/2018/10/18/actualidad/1539847547_146583.html.

BECKER, F. (2018): Campanha de Bolsonaro mente sobre mobilização de mulheres contra o candidato no Facebook. El País Brasil, 17/10. Disponible en: https://brasil.elpais.com/brasil/2018/09/17/politica/1537142202_233134.html.

BENITES, A. (2018): A máquina de 'fake news' nos grupos a favor de Bolsonaro no WhatsApp. El País Brasil, 28/09. Disponible en: https://brasil.elpais.com/brasil/2018/09/26/politica/1537997311_859341.html.

BERALDO, P. (2018): Alvo de fake news sobre Adelio, Manuela d'Ávila é ameaçada nas redes sociais. Estadão, 24/09. Disponible en: https://politica.estadao.com.br/noticias/eleicoes, apos-fake-news-sobre-adeliomanuela-davila-e-ameacada-nas-redes-sociais, 70002516943.

BIMBER, B. (2014): Digital media in the Obama campaigns of 2008 and 2012: Adaptation to the personalized political communication environment. Journal of Information Technology \& Politics, n.11. pp. 130-150.

BIMBER, B. y DAVIS, R. (2003): Campaigning online: the Internet in U.S. elections. Nova York, Oxford University Press, 2003.

BLUMLER, J.G y GUREVITCH, M. (1995): The crisis of public communication. Nova York, Routledge, 1995. 
BLUMLER, J.G. y KAVANAGH, D. (1999): The Third Age of Political Communication: Influences and Features. Political Communication, n.16, pp. 209230.

BRAGA, S. (2014): Eleições como de costume? Uma análise longitudinal das mudanças provocadas nas campanhas eleitorais brasileiras pelos impactos das tecnologias digitais. In: Seminário Mídia, Política e Eleições. São Paulo.

BRAGA, S. y CARLOMAGNO, M. (2018): Elections as usual? longitudinal analysis of the changes caused by digital technologies in Brazilian electoral campaigns (1998-2016). Revista Brasileira de Ciência Política, n. 26, pp. 7-62.

BRITO, F. y MASSARO, H. (2018a): O impulsionamento de conteúdo de précandidaturas na pré-campanha de 2018. Você na Mira - InternetLab - Relatório \#1. Disponible en: http://www.internetlab.org.br/wpcontent/uploads/2018/08/Relat\%C3\%B3rio-1-Voc\%C3\%AA-na-Mira.pdf.

(2018b): Um raio-X do marketing digital dos presidenciáveis. Você na Mira InternetLab - Relatório \#2. Disponible en: http://www.internetlab.org.br/wpcontent/uploads/2018/09/Relat\%C3\%B3rio-Voc\%C3\%AA-na-Mira-2.pdf.

BRITO, F. y VALENTE, M.G. (2018): É hora de se debruçar sobre a propaganda em rede de Bolsonaro. El País Brasil, 21/10. Disponible en: https://brasil.elpais.com/brasil/2018/10/18/opinion/1539892615_110015.html.

CADWALLADR, C. Y GRAHAM-HARRISON, E. (2018): Revealed: 50 million Facebook Profiles harvest for Cambridge Analytica in major data breach. The Guardian. 17 de marzo. Disponible en: https://www.theguardian.com/news/2018/mar/17/cambridge-analytica-facebookinfluence-us-election.

CAMPOS, P. (2018): Empresários bancam campanha contra o PT pelo WhatsApp. Folha S. Paulo, 18/10. Disponible en: https://www1.folha.uol.com.br/poder/2018/10/empresarios-bancam-campanhacontra-o-pt-pelo-whatsapp.shtml.

CANEL, M. y VOLTMER, K. (eds.) (2014): Comparing Political Communication across Time and Space: New Studies in an Emerging Field. Nova York: Palgrave Macmillan.

CBS (2017): Who is Brad Parscale, 27/02. Disponible en: https://www.cbs.com/shows/60_minutes/video/elHhrLFmOS2ZYFqRG68KOPAuO_ aUKPKC/who-is-brad-parscale-l. 
CERVI, E., MASSUCHIN, M. y CARVALHO, F. (orgs) (2016): Internet e eleições no Brasil. Curitiba, CPOP.

DAVIS, R., BAUMGARTNER, J.C., FRANCIA, P.L y MORRIS, J.S. (2009): The internet in the U.S. elections campaigns. In: CHADWICK, A. y HOWARD, P.N. (eds.) Routledge Handbook of Internet Politics. Nova York, Routledge.

DE SÁ, N. (2018): Jair Bolsonaro vai com Bannon, Lula com Sanders. Folha de S. Paulo, 06/10. Disponible en: https://www1.folha.uol.com.br/colunas/nelsondesa/2018/08/jair-bolsonaro-vaicom-bannon-lula-com-sanders.shtml.

DELANY, C. (2017): To Help Elect Trump, Brad Parscale Automated Facebook Best Practices on a Vast Scale. E-politics.com, 11/10. Disponible en: http://www.epolitics.com/2017/10/11/help-elect-trump-parscale-automatedfacebook-best-practices-vast-scale/.

DELMAZO, C. y VALENTE, J.C.L. (2018): Fake news nas redes sociais online: propagação e reações à desinformação em busca de cliques. Media \& Jornalismo, v. 18 (32), pp. 155-169.

DEWEY, J. (2012 [1927]): The Public and Its Problems: An Essay in Political Inquiry. University Park, Pennsylvania, The Pennsylvania University Press.

ENLI, S. y SKOGERBO, E. (2013): Personalized Campaigns in Party-centred Politics: Twitter and Facebook as arenas for political communication. Information, Communication and Society, v. 16 (5).

ESSER, F. y PFETSCH, B. (2004): Comparing Political Communication: Theories, Cases, and Challenges. Cambridge, Cambridge University Press.

(2016): Comparing Political Communication: An Update. National Centre of Competence in Research (NCCR), Challenges to Democracy in the 21st Century, Working Paper n. 89, 2016.

FOX, Z. (2012): The Digital Smackdown: Obama 2008 vs. Obama 2012. Mashable, 23/09. Disponible en: http://mashable.com/2012/09/23/obama-digitialcomparison/\#aYFv3nNDMOqo.

GARRETT, R.K. (2009): Echo Chambers online? Politically motivated selective exposure among Internet news users. Computer-mediated Communication. DOI: 10.1111/j.1083-6101.2009.01440.x.

GOMES, W. (2018): A democracia no mundo digital: História, problemas e temas. São Paulo, SESC. 
GOMES, W. y AGGIO, C. (2009): Campanhas Online: O percurso de formação das questões, problemas e configurações a partir da literatura produzida entre 1992 e 2009. I Seminário Nacional Sociologia e Política UFPR, Curitiba.

GOMES, W., FERNANDES, B., REIS, L. y SILVA, T. (2009): Politics 2.0: a campanha on-line de Barack Obama em 2008. Revista de Sociologia Política, Curitiba, v. 17 (34), pp.29-43.

GRAHAM, T., BROERSMA, M., HAZELHOFF, K. y HAAR, G.V. (2013): Between Broadcasting Political Messages and Interacting With Voters: the use of Twitter during the 2010 UK general election campaign. Information, Communication \& Society, V. 16.

GRASSEGGER, H. y KROGERUS, M. (2017): The data that turned the world upside down. Motherboard, 28/01. Disponible en: https://motherboard.vice.com/en_us/article/mg9vvn/how-our-likes-helped-trumpwin.

GUREVITCH, M. y BLUMLER, J.G. (2004): State of the Art of Comparative Political Communication Research: Poised for Maturity. In: ESSER, F. y PFETSCH, B. (eds.) Comparing Political Communication: Theories, Cases, and Challenges. Cambridge, Cambridge University Press.

HALLIN, D.C y MANCINI, P. (2011): Comparing Media Systems: Beyond the Western World. Cambridge, Cambridge University Press.

(2004a): Comparing Media Systems: Three Models of media and politics. Cambridge, Cambridge University Press.

(2004b): Americanization, Globalization, and Secularization: Understanding the Convergence of Media Systems and Political Communication. In: ESSER, F. y PFETSCH, B. Comparing Political Communication: Theories, Cases, and Challenges. Cambridge, Cambridge University Press.

HOWARD, P.N. (2006): New Media Campaigns and the Managed Citizen. Cambridge: Cambridge University Press.

INTELLIGENCE COMMUNITY ASSESSMENT (2017): Assessing Russian Activities and Intentions in Recent US Elections. Office of the Director of National Intelligence. Disponible

https://www.dni.gov/files/documents/ICA_2017_01.pdf.

ITUASSU, A., CAPONE, L, PARENTE, T. y PECORARO, C. (2014): Internet, eleições e democracia: o uso das redes sociais digitais por Marcelo Freixo na 
campanha de 2012 para a Prefeitura do Rio de Janeiro. Compolítica, v. 2 (4), pp. 59-86.

ITUASSU, A., LIFSCHITZ, S., CAPONE, L. y MANNHEIMER, V. (2018): "Politics 3.0"? De @realDonaldTrump para as eleições de 2018 no Brasil. In: Anais do XXVII Encontro Anual da Compós, Belo Horizonte: Compós.

KING, G., KEOHANE, R.O. y VERBA, S. (1994): Designing Social Inquiry: Scientific Inference in Qualitative Research. Princeton, Princeton University Press.

LE DUC, L., NIEMI, R.G. y NORRIS, P. (2002): Comparing Democracies 2: New challenges in the study of elections and voting. London, Sage Publications.

LIPPMANN, W. (2010 [1921]): Public Opinion: A Classic in Political and Social Thought. New York: Feather Trail Press.

MARQUES, F.P.J.A., SAMPAIO, R.C. y AGGIO, C. (orgs.) (2013): Do clique à urna: Internet, redes sociais e eleições no Brasil. Salvador, EDUFBA.

MIOLA, E. y MARQUES, F.P.J. (2018): Razão e emoção nas estratégias eleitorais: a campanha à prefeitura de Curitiba em 2016 no Facebook. In: Anais do XXVII Encontro Anual da Compós, Belo Horizonte, Compós.

MOTA, C.V. (2017): Robôs e 'big data': as armas do marketing político para as eleições de 2018. BBC Brasil. Disponible en: http://www.bbc.com/portuguese/brasil-41328015

MOTA, M., COUTO, M. y ROCHA, G. (2018): Mensagens com conteúdo \#FAKE sobre fraude em urnas eletrônicas se espalham nas redes. O Globo, 06/10. Disponible en: https://oglobo.globo.com/fato-ou-fake/mensagens-com-conteudofake-sobre-fraude-em-urnas-eletronicas-se-espalham-nas-redes-23134205.

MUÑIZ, C., DADER, J.L., TÉLLEZ, N.M. y SALAZAR, A. (2016): ¿Están los políticos políticamente comprometidos? Análisis del compromiso político 2.0 desarrollado por los candidatos a través de Facebook. Cuadernos.Info, n. 39, pp. 135-150. DOI: $10.7764 /$ cdi.39.970.

MURTA, F., LEO, L., ITUASSU, A., LA ROVERE, R. y CAPONE, L. (2017): Eleições e mídias sociais: Interação e participação no Facebook durante a campanha para a Câmara dos Deputados em 2014. Compolítica, v. 7(1), pp. 47-72.

NIELSEN, R. K. y VACCARI, C. (2013): Do People "Like" Politicians on Facebook? Not Really. Large-Scale Direct Candidate-to-Voter Online Communication as an Outlier Phenomenon. International Journal of Communication v.7, pp.2333-2356. 
NORRIS, P. (2000): A Virtuous Circle Political communications in postindustrial societies. Cambridge, Cambridge University Press.

(2002): Campaign Communications. En LE DUC, L., NIEMI, R.G. y NORRIS, P. (orgs.). Comparing Democracies 2: New challenges in the study of elections and voting. London, Sage Publications.

NORRIS, P. (2009): Comparative Political Communications: Common Frameworks or Babelian Confusion? Government and Opposition, v. 44 (3), pp. 321-340. DOI: 10.1111/j.1477-7053.2009.01290.x

(2019): Cultural Backlash: Trump, Brexit, and Authoritarian Populism. London, Cambridge University Press.

PAMPLONA, M. y STUVEN, A.M. (2010): Estado e Nação no Brasil e no Chile ao longo do século XIX. Rio de Janeiro, Garamond/Faperj.

PERSILY, N. (2017): Can democracy survive the Internet? Journal of Democracy, v. 28(2).

QUATTROCIOCCHI, W., SCALA, A. y SUNSTEIN, C.R. (2016): Echo Chambers on Facebook, Disponible en SSRN: https://ssrn.com/abstract=2795110.

ROGERS, M. L. (2012): Revisiting The Public and Its Problems. En: DEWEY, J. The Public and Its Problems: An Essay in Political Inquiry. University Park, Pennsylvania, The Pennsylvania University Press.

RUEDIGER, M.A. (2017): Robôs, redes sociais e política no Brasil. DAPP/FGV-Rio. Disponible en: http://dapp.fgv.br/robos-redes-sociais-e-politica-estudo-dafgrdapp-aponta-interferencias-ilegitimas-no-debate-publico-na-web/.

(2018a): Reação a boatos superou a difusão de informações contra Marielle no Twitter. DAPP/FGV-Rio. Disponible en: http://dapp.fgv.br/reacao-boatossuperou-difusao-de-informacoes-contra-marielle-no-twitter-aponta-estudo-da-fgvdapp/.

(2018b): Robôs, redes sociais e política no Brasil: Análise de interferências de perfis automatizados nas eleições de 2014. DAPP/FGV-Rio. Disponible en: http://dapp.fgv.br/robos-redes-sociais-e-politica-estudo-da-fgvdapp-apontainterferencias-ilegitimas-no-debate-publico-na-web/.

(2018c): Redes sociais nas eleições 2018. DAPP/FGV-Rio. Disponible en: https://observa2018.com.br/wp-content/uploads/2018/10/Redes-Sociais-nasElei\%C3\%A7\%C3\%B5es18_corrigido.pdf. 
(2018d): A semana nas redes: 18/10/2018. DAPP/FGV-Rio. Disponible en: https://observa2018.com.br/wp-content/uploads/2018/10/18-10-Dapp-

Report.pdf.

RUTLEDGE, P. (2013): How Obama Won the Social Media Battle in the 2012 Presidential Campaign. The Media Psychology Blog. Disponible en: http://mprcenter.org/blog/2013/01/how-obama-won-the-social-media-battle-inthe-2012-presidential-campaign/.

SALGADO, D. (2018): Livro citado por Bolsonaro no Jornal Nacional não foi distribuído em escola. O Globo, 29/10. Disponible en: https://oglobo.globo.com/brasil/livro-citado-por-bolsonaro-no-jornal-nacionalnao-foi-distribuido-em-escola-23021610.

SCHERER, M. (2012): Inside the Secret World of the Data Crunchers Who Helped Obama Win. Time, 19/11. Disponible en: http://swampland.time.com/2012/11/07/inside-the-secret-world-of-quants-anddata-crunchers-who-helped-obama-win/.

SOUSA, I. y MARQUES, F.P.J. (2017): Campanha negativa e formas de uso do Facebook nas eleições presidenciais brasileiras em 2014. MOREIRA, A., ARAÚJO, E. y SOUSA, H. (orgs.) Comunicação, contexto e política: tempos, contextos e desafios. Braga: CECS - Centro de Estudos de Comunicação e Sociedade Universidade do Minho.

STROMMER-GALLEY, J. (2000): On-line interaction and why candidates avoid it. Journal of Communication, v. 50 (4), pp. 111-132.

(2019): Presidential Campaigning in the Internet Age. New York, Oxford University Press.

SWANSON, D.L. y MANCINI, P. (1996): Politics, Media, and Modern Democracy: An International Study of Innovations in Electoral Campaigning and Their Consequences. Westport (Conn.): Praeger.

SYMANTEC (2016). Internet Security Threat Report. V.21, April. Disponible en: https://www.symantec.com/content/dam/symantec/docs/reports/istr-21-2016en.pdf.

VACCARI, C. (2008): From the air to the ground: the internet in the 2004 US presidential campaign. New Media \& Society, DOI: 10.1177/1461444808093735.

VACCARI, C., VALERIANI, A., BARBERÁ, P., JOST, J., NAGLER, J. y TUCKER, J. (2016): Of Echo Chambers and Contrarian Clubs: Exposure to Political 
Disagreement Among German and Italian Users of Twitter. Social Media \& Society, 2 (3). DOI: 10.1177/2056305116664221V.

VOLTMER, K. (2006): Mass Media and Political Communication in New Democracies. Nova York, Routledge.

WOOLLEY, S.C. y GUILBEAULT, D. (2019): United States: Manufacturing Consensus Online. In WOOLLEY, S.C.; HOWARD, P.N. (eds.) Computational Propaganda: Political Parties, Politicians, and Political Manipulation on Social Media. Oxford, Oxford University Press.

(2017): Computational Propaganda in the United States of America: Manufacturing Consensus Online. Computational Propaganda Research Project, Working paper n. 2017.5.

WOOLLEY, S.C. y HOWARD, P.N. (eds.) (2019): Computational Propaganda: Political Parties, Politicians, and Political Manipulation on Social Media. Oxford, Oxford University Press.

ZIELONKA, J. (2015): Media and Politics en New Democracies Europe in a Comparative Perspective. Oxford, 2015. 